Revista Búsqueda, Vol. 4 Núm. 19:149-157; 2017. ISSN: 0123-9813

DOI: doi.org/10.21892/01239813.368

ORIGINAL

\title{
Consumo máximo de oxígeno, frecuencia cardíaca máxima y velocidad aeróbica máxima de árbitros colombianos de fútbol
}

\author{
Maximum oxygen consumption, maximum heart rate and maximum \\ aerobic speed of Colombian soccer referees
}

Brian Johan Bustos-Viviescas ${ }^{1}$, Andrés Alonso Acevedo-Mindiola ${ }^{2}$, José Alexander Ortiz-Novoa ${ }^{3}$

${ }^{1}$ Estudiante de Educación Básica con énfasis en Educación Física, Recreación y Deportes en la Universidad de Pamplona, Colombia. Docente en Educación Secundaria. Identificador de autor: https://orcid. org/0000-0002-4720-9018

${ }^{2}$ Licenciado en Educación Básica con énfasis en Educación Física, Recreación y Deportes en la Universidad de Pamplona, Colombia. Docente en Educación Secundaria. Identificador de autor: https://orcid.org/00000003-0125-7265; autor corresponsal: andresacevedo26@hotmail.com

${ }^{3}$ Estudiante de Educación Básica con énfasis en Educación Física, Recreación y Deportes en la Universidad de Pamplona, Colombia. Árbitro profesional de la Federación

Recibido: 24-07-2017; Revisado: 09-08-2017; Aceptado: 27-09-2017

\section{Resumen}

El árbitro de fútbol tiene un rol importante durante un juego, por lo cual debe tener una adecuada aptitud física que le permita un óptimo rendimiento físico en el arbitraje. Por tal motivo, el objetivo de este estudio fue determinar el consumo máximo de oxígeno (Vo2máx), frecuencia cardíaca máxima (FCM) y velocidad aeróbica máxima (VAM) de árbitros colombianos de fútbol (ACF) a 320 metros sobre el nivel del mar (320msnm). Se realizó una investigación de enfoque cuantitativo con un diseño de campo y una muestra a conveniencia participando voluntariamente doce árbitros entre los 20 y 33 años distribuidos en grupos de cuatro por cada categoría: A, B y C. Se les aplicó el Test Yo-Yo de resistencia nivel 1. El test fue aplicado en la ciudad de Cúcuta, ubicada a $320 \mathrm{msnm}$. Los datos fueron analizados en el paquete estadístico IBM SPSS V. 22, con un nivel de confianza del $95 \%$ y un p-valor de 0,05, en el cual se realizó una estadística no paramétrica y se utilizó la prueba de la U de Mann-Whitney.

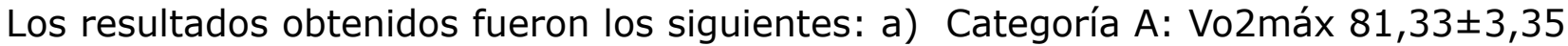

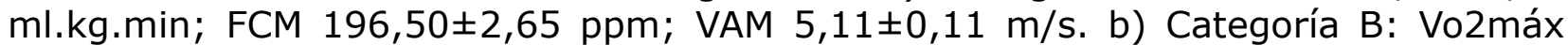

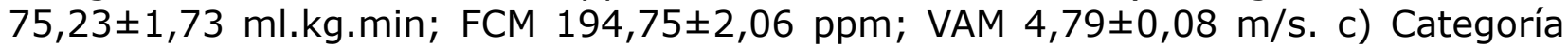

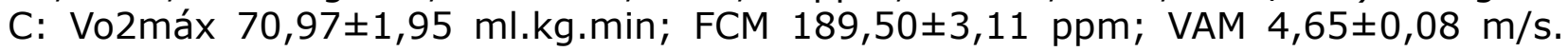
Estos datos evidenciaron diferencias estadísticamente significativas al comparar estas variables $(p<0,05)$. En conclusión existen diferencias estadísticamente significativas entre el Vo2máx, FCM y VAM de ACF a 320msnm $(p<0,05)$.

Palabras clave: consumo máximo de oxígeno, frecuencia cardiaca máxima, velocidad aeróbica máxima. 


\section{Abstract}

The soccer referee has an important role during a game, for which you must have an adequate physical aptitude that allows an optimal physical performance in the arbitration. For that reason, the objective of this study, was to determine the maximum consumption of oxygen (Vo2max), maximum heart rate (MHR) and maximum aerobic speed (MAS) of Colombian soccer referees (CSF) at 320 meters on the level of the sea (320m/s). An investigation of quantitative approach with a design of field and a sample of convenience voluntarily participating twelve referees, between 20 and 33 years old, voluntarily participated. They had distributed in groups of four by category: $A, B$ and $C$. The Yo-Yo Test of resistance level 1 was applied to them, after signing an informed consent which specified the purpose of the study, the description of the test and its risks and benefits. The test was applied in the city of Cucuta, located at $320 \mathrm{~m} / \mathrm{s}$. The data were analyzed in the statistical package IBM SPSS V. 22, with a 95\% confidence level and a $p$-value of 0.05 , in which was a non-parametric statistic, and the Mann-Whitney $U$ test was used. These are the results obtained by categories: a)

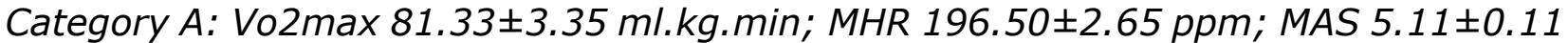

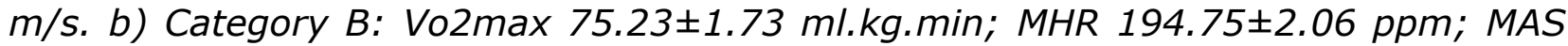

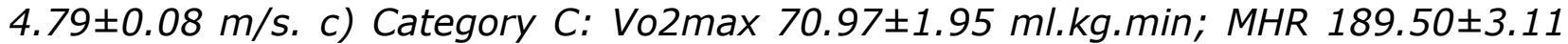
ppm; MAS 4.65 $\pm 0.08 \mathrm{~m} / \mathrm{s}$. The results showed statistically significant differences when comparing these variables $(p<0.05)$. In conclusion, there are statistically significant differences between the Vo2max, MHR and MAS to CFS at 320m/s $(p<0.05)$.

Key words: maximum consumption of oxygen, maximum heart rate, maximum aerobic speed.

\section{Introducción}

Actualmente, cualquier profesional de las ciencias de la actividad física y deportiva puede reconocer al fútbol como un deporte físicamente muy agotador (Fernández, Da Silva y Arruda, 2008). Los investigadores han demostrado que los rendimientos físicos de los árbitros están interrelacionados con los de los jugadores durante el mismo partido (Weston et al., 2012), por ello, el árbitro de nivel elite está expuesto a demandas físicas similares a las que se encuentran en un futbolista en el juego (Mazaheri, Halabchi, Barghi y Mansournia, 2016), de manera que debe ser capaz de sobrellevar las condiciones de juego impuestas por los jugadores (Yanci et al., 2014) para poder controlar el comportamiento de estos y aplicar las reglas del juego durante el fútbol competitivo (Abass et al., 2011). Los árbitros juegan un papel fundamental en el fútbol (Ruiz et al., 2011), dado a que deben mantenerse al tanto del juego en todo momento, para asegurar un posicionamiento óptimo en la toma de decisiones (Weston et al., 2012), y para ello se requiere de un buen rendimiento físico (Fernández, Da Silva y Arruda, 2008; Mazaheri, Halabchi, Barghi y Mansournia, 2016).

Durante un partido de fútbol, se exige a los árbitros una alta capacidad aeróbica, por la constante variación de recorridos con descansos cortos a intensidades moderadas (Castagna, Grant, y D'Ottavio, 2007), puesto que los sistemas metabólicos predominantes requeridos en el partido de fútbol son los de respuestas aeróbicas y metabólicas, similares a las encontradas en ejercicio de resistencia (Reilly, Bangsbo y Franks, 2000), por 
ende, el Vo2máx es un indicador fiable del rendimiento en árbitros, puesto que, como indican Arecheta, Gómez y Lucía (2006), el Vo2máx puede contribuir al rendimiento físico en el fútbol, en vista de que una alta capacidad cardiorrespiratoria será capaz de mantenerse en el juego a un ritmo mayor todo el tiempo. Por ende, un indicador fiable para la dosificación de la carga de entrenamiento aeróbico sobre todo a intensidades superiores al $85 \%$ del VO2máx puede ser la velocidad aeróbica máxima (Leger, 1999; Billat et al, 1999; Tuimil y Rodríguez, 2000 y 2001, citados por Díaz, 2015). Asimismo, algunos estudios muestran que los árbitros de fútbol de alto nivel pueden alcanzar el $85 \%-95 \%$ de su frecuencia cardíaca máxima, en un partido completo (Gamboa, Forero, Urbina y Mendoza, 2008).

La literatura científica se enfoca en el entrenamiento a altitud media y alta para observar los cambios fisiológicos que se originan en los deportistas frente a la hipoxia producida por la disminución de la presión parcial del oxígeno en el aire inspirado, sin embargo, actualmente no existen estudios sobre el consumo máximo de oxígeno, frecuencia cardíaca máxima y velocidad aeróbica máxima de árbitros colombianos de fútbol a 320 metros sobre el nivel del mar teniendo la categoría a la que pertenecen. En vista de ello, no se tiene información de las características físicas que tienen los sujetos que ejercen dicha labor al igual que se desconoce si existen diferencias por categoría, razón por la cual se planteó este estudio, el cual tuvo como objetivo determinar dichos datos.

\section{Metodología}

El estudio presento una investigación de enfoque cuantitativo con un diseño de campo, en el cual se recolectan los datos directamente de los sujetos investigados sin controlar alguna variable (Arias, 2012). Además, se aplicó una muestra a conveniencia puesto que la selección se realizó dada la conveniente accesibilidad y proximidad de los sujetos para los investigadores (Scribano, 2007).

\section{Participantes}

En este estudio participaron voluntariamente doce árbitros entre los 20 y 33 años, distribuidos por la categoría a la que pertenecían. De esta manera los árbitros de la categoría A son los de mayor experiencia en el arbitraje, los de $B$ con una menor experiencia que los de $A$, y por último los de categoría $C$ de menor experiencia. Se obtuvieron cuatro árbitros para la categoría $\mathrm{A}$, cuatro para la B y cuatro para la $\mathrm{C}$. Todos firmaron un consentimiento informado en el que se especificaba el objetivo del estudio, la descripción del test y los riesgos del mismo, así como los beneficios por participar del estudio. Cabe agregar que este estudio siguió las directrices establecidas en la Declaración de Helsinki (Asociación Médica Mundial, 2013) al igual que los estándares éticos establecidos para investigaciones en ciencias del deporte y del ejercicio (Harriss y Atkinson, 2013).

\section{Procedimiento}

El estudio fue realizado en la plaza de banderas de la ciudad de Cúcuta, ubicada a $320 \mathrm{msnm}$, y en horas de la tarde (4:00 PM). Los valores de peso y talla fueron tomados con la menor cantidad de ropa y sin calzado. Posteriormente se realizó el Test Yo-Yo de resistencia nivel 1, el cual ha demostrado una buena concordancia con la ergoespirometría directa en tapiz rodante (Longo, Aquilino, Cardey y Lentini, 2017). Este test consiste en realizar carreras de ida y vuelta sobre una distancia de veinte metros, en las cuales el tiempo para recorrerla estará indicado por medio de una señal sonora (bips), y su velocidad aumenta progresivamente 
hasta alcanzar el agotamiento. La ecuación utilizada fue la propuesta por Bangsbo, Iaia y Krustrup (2008), la cual es específica para el nivel del test empleado.

Ecuación Yo-Yo IR 1 test

Vo2 Máx (ml/ $\mathrm{kg} / \mathrm{min})$ : Distancia $(\mathrm{m}) \times$ $0,0084 \times 36,4$

\section{Instrumentos para la recolección de datos}

Para el desarrollo del estudio se utilizaron los siguientes equipos para la recolección de datos.

- Báscula modelo PCE-TS 150: utilizada para obtener el peso de los árbitros con un rango de pesado de hasta 150 $\mathrm{kg}$.

- Pulsómetro Polar M 600 tecnología Android Wear 2.0: utilizado para obtener la frecuencia cardiaca de los sujetos.

- Tallímetro ref. 216 marca Seca: utilizado para medir la talla de los sujetos.

- Magnetófono tc530: empleado para reproducir la grabación del Test Yo-Yo de resistencia.

- Conos: utilizados para demarcar el recorrido cada veinte metros.

\section{Análisis de datos}

Todos los datos fueron tabulados y analizados en el paquete estadístico IBM SPSS V. 22, en el cual se aplicó una estadística no paramétrica dada la pequeña muestra del estudio. El nivel de confianza fijado fue del $95 \%$ y el p-valor de 0,05, para poder comparar las variables y establecer diferencias significativas entre categorías por medio de la prueba de la U de Mann-Whitney.

\section{Resultados}

En la Tabla 1 se exponen las medias y desviaciones estándar de edad, peso, talla y experiencia arbitral de las categorías de los participantes del estudio, se puede percibir de forma incremental la experiencia en años de arbitraje de la categoría $C$ a la $A$, por lo cual los que pertenecen a categorías más altas tienen más tiempo en el arbitraje del fútbol.

Por su parte, la frecuencia de entrenamiento y duración de la sesión, así como también la frecuencia de arbitraje semanal y mensual se muestra en la Tabla 2. Por otro lado, la media de frecuencia de entrenamiento y duración de la sesión de la categoría $C$ es menor con respecto a las categorías $\mathrm{A}$ y $\mathrm{B}$.

Tabla 1. Características generales

\begin{tabular}{ccccccccc}
\hline \multirow{2}{*}{ Categoría } & \multicolumn{2}{c}{ Edad (años) } & \multicolumn{2}{c}{ Peso (kg) } & \multicolumn{2}{c}{ Talla (m) } & \multicolumn{2}{c}{ Exp. Arbitral (años) } \\
& Media & Desv. Est. & Media & Desv. Est. & Media & Desv. Est. & Media & Desv. Est. \\
\hline A (4) & 27,50 & 2,38 & 70,00 & 6,78 & 1,72 & 0,07 & 11,50 & 3,32 \\
B (4) & 22,50 & 1,29 & 75,00 & 4,55 & 1,84 & 0,05 & 7,00 & 1,63 \\
C (4) & 21,25 & 1,26 & 65,75 & 3,30 & 1,75 & 0,05 & 2,75 & 1,71 \\
\hline
\end{tabular}


Bustos-Viviescas et al. Consumo máximo de oxígeno de árbitros colombianos de fútbol

Tabla 2. Distribución de las sesiones de entrenamiento y arbitraje de partidos

\begin{tabular}{ccccccccc}
\hline & \multicolumn{2}{c}{ Frecuencia de } & \multicolumn{2}{c}{ Duración de la } & \multicolumn{2}{c}{ Frecuencia de } & \multicolumn{2}{c}{ Frecuencia de } \\
Categoría & \multicolumn{2}{c}{ entrenamiento } & \multicolumn{2}{c}{$\begin{array}{c}\text { sesión (h) } \\
\text { arbitraje semanal }\end{array}$} & \multicolumn{2}{c}{ arbitraje mens ual } \\
& Media & Desv. Est. & Media & Desv. Est. & Media & Desv. Est. & Media & Desv. Est. \\
\hline A (4) & 5,00 & 0,00 & 2,00 & 0,00 & 2,00 & 0,00 & 8,00 & 0,00 \\
B (4) & 4,25 & 0,96 & 2,00 & 0,00 & 2,25 & 0,50 & 8,50 & 1,00 \\
C (4) & 3,50 & 1,00 & 1,00 & 0,00 & 2,25 & 0,50 & 8,75 & 0,96 \\
\hline
\end{tabular}

Teniendo en cuenta los resultados obtenidos en el test, presentados en la Tabla 3 y Tabla 4 , se puede identificar que la frecuencia cardíaca máxima alcanzada por los árbitros de la categoría C presenta diferencias estadísticamente significativas con relación a las categorías A y $B(p<0,05)$. El periodo final alcanzado presentó diferencia significativa al comparar todas las categorías, lo cual indica que las categorías más altas soportaron mayores periodos en el test, y también esto se representa con un mayor volumen máximo de oxigeno $(p<0,05)$, mientras que la velocidad aeróbica máxima solo obtuvo diferencias significativas al comparar la categoría $A$ con $B$ y $A$ con $C(p<0,05)$, por ende, se observa que las velocidades aeróbicas obtenida en el test por parte de los árbitros de las categorías $\mathrm{B}$ y $\mathrm{C}$ no se diferencias significativamente $(p>0,05)$.

Tabla 3. Variables del test

\begin{tabular}{ccccccccc}
\hline \multirow{2}{*}{ Categoría } & \multicolumn{2}{c}{ FC Máx (ppm) } & \multicolumn{2}{c}{ Periodo final } & \multicolumn{2}{c}{ Vo2Máx } & \multicolumn{2}{c}{ VAM } \\
& Media & Desv. Est. & Media & Desv. Est. & Media & Desv. Est. & Media & Desv. Est. \\
\hline A (4) & 196,50 & 2,65 & 20,50 & 0,84 & 81,33 & 3,35 & 5,11 & 0,31 \\
B (4) & 194,75 & 2,06 & 18,98 & 0,39 & 75,23 & 1,73 & 4,79 & 0,08 \\
C (4) & 189,50 & 3,11 & 17,85 & 0,49 & 70,97 & 1,95 & 4,65 & 0,08 \\
\hline
\end{tabular}

Tabla 4. Comparación de medias de las variables del test

\begin{tabular}{|c|c|c|c|c|}
\hline \multirow{2}{*}{ Variables } & \multirow{2}{*}{$\begin{array}{l}\text { U de Mann- } \\
\text { Whitney }\end{array}$} & \multicolumn{3}{|c|}{ Categorías } \\
\hline & & A-B & B-C & A-C \\
\hline \multirow{2}{*}{ FC Máx (ppm) } & $\mathrm{Z}$ & $-1,16$ & $-2,03$ & $-2,18$ \\
\hline & Sig. Asintónica (p) & 0,24 & $0,04 *$ & 0,03 * \\
\hline \multirow{2}{*}{ Periodo final } & Z & $-2,18$ & $-2,31$ & $-2,31$ \\
\hline & Sig. Asintónica (p) & $0,03 *$ & $0,02^{*}$ & $0,02^{*}$ \\
\hline \multirow{2}{*}{ Vo2Máx } & Z & $-2,18$ & $-2,31$ & $-2,31$ \\
\hline & Sig. Asintónica (p) & $0,03 *$ & $0,02 *$ & $0,02 *$ \\
\hline \multirow{2}{*}{ VAM } & Z & $-2,09$ & $-1,87$ & $-2,35$ \\
\hline & Sig. Asintónica (p) & $0,04 *$ & 0,06 & $0,02 *$ \\
\hline
\end{tabular}

Leyenda: *Diferencia significativa entre las medias $(p<0,05)$ 


\section{Discusión}

El objetivo del presente estudio fue determinar el consumo máximo de oxígeno, frecuencia cardíaca máxima y velocidad aeróbica máxima de árbitros colombianos de fútbol a $320 \mathrm{msnm}$. Entre los principales hallazgos se evidenció diferencia estadísticamente significativa entre la frecuencia cardiaca máxima obtenida en el test al comparar las categorías $(p<0,05)$; esto se debe principalmente a que el nivel de entrenamiento está relacionado con esta variable (Whyte et al., 2008; Zavorsky, 2000), por ende, los participantes de las categorías A y B presentan una mayor frecuencia cardíaca máxima en este estudio con respecto a los de la categoría $\mathrm{C}$.

Por otro lado, en relación a la prescripción de la intensidad del esfuerzo, quizás el componente de la carga que en mayor medida va a orientar y condicionar las adaptaciones cardiorrespiratorias es la velocidad aeróbica máxima (Pallarés y Morán-Navarro, 2012), dado a que esta representa la base fundamental para organizar de forma precisa las cargas de trabajo aeróbico durante el entrenamiento (Campillo, Nkuignia y Matías, 2013), por lo cual es un índice valido para evaluar el rendimiento aeróbico de los atletas, en vista de que indica la dimensión de la intensidad a la que se debe ajustar el volumen que constituye la resistencia específica (Campillo, Nkuignia y Matías, 2013). Sin embargo, en este estudio solamente se evidenció diferencia estadísticamente significativa con los árbitros más experimentados de la categoría $A$ en comparación con los de la categoría $\mathrm{B}$ y $\mathrm{C}$, mientras que el volumen máximo de oxígeno fue significativamente diferente entre todas las categorías, dado al periodo final alcanzado por los participantes, por lo cual se debe ser precavido al usar indiscriminadamente ambas variables como iguales en la evaluación del rendimiento aeróbico de árbitros.

Cabe destacar que este estudio fue realizado a $320 \mathrm{msnm}$, por lo tanto los participantes no sufrieron ninguna modificación fisiológica en reposo ni se vieron afectados en el rendimiento físico (Moreno, 2001). En un estudio realizado por Mercado, Sánchez y Gutiérrez (2015) se evidenció una disminución del volumen máximo de oxígeno y velocidad aeróbica máxima al comparar el protocolo realizado en alturas bajas y medias, puesto que la disminución de la presión parcial del oxígeno en el aire inspirado disminuye el volumen máximo de oxígeno y con esto la velocidad aeróbica máxima y la frecuencia cardíaca máxima también. A su vez, los resultados superiores en los árbitros más experimentados son similares a los del estudio realizado por Casajus y Castagna (2007), en el cual estos autores concluyen que los árbitros más antiguos son capaces de alcanzar los niveles físicos de acondicionamiento físico que se han sugerido que son apropiados para hacer frente a las demandas de los partidos.

\section{Conclusiones}

Teniendo en cuenta los hallazgos de este estudio, se concluye que existen diferencias estadísticamente significativas entre el consumo máximo de oxígeno, frecuencia cardíaca máxima y velocidad aeróbica máxima de árbitros colombianos de fútbol a 320 msnm teniendo en cuenta la categoría a la que pertenecen $(p<0,05)$. De esta manera, se destaca que los árbitros que tienen una mayor experiencia, pertenecientes a la categoría $A$, tendrán un óptimo rendimiento físico que favorece en el éxito del arbitraje. Cabe mencionar, que conociendo los datos determinados en este estudio, servirán como punto de partida para la planificación y control del entrenamiento de las cargas con los árbitros pertenecientes a las demás categorías. 


\section{Referencias bibliográficas}

Abass, A. O., Moses, M. O., Alabi, E. F., Adedugbe, B. O., Falola, K. O., Y Abayomi, A. O. (2011). Relationships between bio-physiological parameters and speed performance of Nigerian FIFA referees. Journal of Physical Education and Sport Management, 2(6), 58-61.

Arecheta Pérez, C., Gómez López, M. y Lucía Mulas, A. (2006). La importancia del vo2max para realizar esfuerzos intermitentes de alta intensidad en el fútbol femenino de élite. Kronos, 5(9), 4-12. Recuperado de: https://dialnet.unirioja.es/servlet/ articulo? codigo $=2042032 \mathrm{https}: / /$ dialnet.unirioja.es/servlet/ articulo? codigo $=2042032$

Arias, F. (2012). El Proyecto de investigación. Introducción a la metodología científica. Caracas, Venezuela: EDITORIAL EPISTEME, C. A.

Asociación Médica Mundial. (2013). Declaración de Helsinki de la AMM-Principios éticos para las investigaciones médicas en seres humanos. Recuperado de: http:// www.isciii.es/ISCIII/es/contenidos/ $\mathrm{fd}$-investigacion/fd-evaluacion/ fd-evaluacion-etica-investigacion/ Declaracion-Helsinki-2013-Esp. pdfhttp://www.isciii.es/ISCIII/ es/contenidos/fd-investigacion/ $\mathrm{fd}$-evaluacion/fd-evaluacion-eticainvestigacion/Declaracion-Helsinki2013-Esp.pdf

Bangsbo, J., Iaia, F. M. y Krustrup, P. (2008). The Yo-Yo intermittent recovery test. Sports medicine, $38(1), 37-51$.
Billat, V., Bernard, O., Pinoteau, J., Petit, B., y Koralstein, J.P. (1994). Time to exhaustion at VO2 Máx and lactate steady velocity in subelite longdistance runner. Archives international oh Physiology. Biochemistry and Biophysique, 102(4) 215219. doi:. http://dx.doi. org/10.3109/13813459409007541

Campillo, P., Nkuignia, O. y Matías, C. (2013). Pruebas de velocidad aeróbica máxima conjóvenes futbolfutbolistas. Control y programación de la intensidad de los entrenamientos. Apunts. Educación Física y Deportes, 113, 45-51. Doi: http://dx.doi. org/10.5672/apunts.2014-0983. es.(2013/3).113.04http://dx.doi. org/10.5672/apunts.2014-0983. es.(2013/3).113.04

Casajus, J. A., y Castagna, C. (2007). Aerobic fitness and field test performance in elite Spanish soccer referees of different ages. Journal of Science \&y Medicine in Sport, 10(6), 382-389. Doi: http://dx.doi. org/10.1016/j.jsams.2006.08.004

Castagna, C., Grant, y D'Ottavio, S. (2007). Physiological aspects of soccer refereeing performance and training. Sports Medicine, 37(7), 625-646. Recuperado de: https://www.researchgate. net/publication/256613906 Physiological_Aspects_of_Soccer_ Refereeing_Performance_and_ Traininghttps://www. researchgate. net/publication/256613906 Physiological_Aspects_of_Soccer_ Refereeing_Performance_and_ Training 
Díaz, D. A. (2015). Tiempo límite a la velocidad asociada al consumo máximo de oxígeno en jóvenes atletas del municipio de Chía, Colombia. Lecturas: Educación Física y Deportes, 20(206). Recuperado de: http://www.efdeportes.com/efd206/ velocidad-asociada-al-consumomaximo-de-oxigeno.htmhttp:// www.efdeportes.com/efd206/ velocidad-asociada-al-consumomaximo-de-oxigeno.htm

Fernández Vargas, G. E., Dda Silva, A. I., y Arruda, M. (2008). Perfil aAntropométrico y aAptitud fFísica de áÁrbitros del fFútbol pProfesional cChileno. International Journal of Morphology, 26(4), 897-904. Doi: http://dx.doi.org/10.4067/S071795022008000400019.

Gamboa Carmona, W. O., Forero Delgado, M. A., Urbina Bonilla, A. P. y Mendoza Romero, D. (2008). Comparación de dos pruebas de resistencia aeróbica continua e intermitente en condiciones de altura intermedia en árbitros de fútbol. Revista Corporeizando, 1(2), 38-49.

Harriss, D., \&y Atkinson, G. (2013). Ethical standards in sport and exercise science research: 2014 update. International Journal of Sports Medicine, 34(12), 1025-1028. Doi: http://dx.doi. org/10.1055/s-0033-1358756

Longo, A. F., Aquilino, G. D., Cardey, M. L. y Lentini, N. A. (2017). VO 2 max assessment in athletes: aA thorough method comparison study between Yo-Yo test and direct measurement. Apunts Med Esport, 52(193), 17-22. Doi: http://dx.doi.org/10.1016/j. apunts.2016.07.001
Mazaheri, R., Halabchi, F., Barghi, T. S., y Mansournia, M. A. (2016). Cardiorespiratory Fitness and Body Composition of Soccer Referees; Do These Correlate with Proper Performance? Asian Journal of Sports Medicine, 7(1). Doi: https://dx.doi. org/10.5812\%2Fasjsm. 29577

Mercado Ruíz, H. A., Sánchez Rodríguez, D. A. y Gutiérrez, J. (2015). Comportamiento de los niveles del vo2 máximo en futbolistas prejuveniles en diferentes altitudes. Revista digital: Actividad Física y Deporte, 1(2), 5-21. Recuperado de: http://www.udca.edu.co/wpcontent/uploads/revista-deportes/ revista-digital-actividad-fisicadeporte-vol1-no2.pdfhttp://www. udca.edu.co/wp-content/uploads/ revista-deportes/revista-digitalactividad-fisica-deporte-vol1-no2. pdf

Moreno Suárez, E. L. (2001). Preparación del deportista para el entrenamiento de altura. Lecturas: Educación Física y Deporte, 7(40). Recuperado de: http://www.efdeportes.com/efd40/ altura.htmhttp://www.efdeportes. com/efd40/altura.htm

Pallarés, J. G. y Morán-Navarro, R. (2012). Propuesta metodológica para el entrenamiento de la resistencia cardiorrespiratoria. Journal of Sport and Health Research, 4(2), 119-136. Recuperado de: http://journalshr. com/papers/Vol\%204_N\%202/ V04_2_3.pdf

Reilly, T., Bangsbo, J., \& Franks, A. (2000). Anthropometric and physiological predispositions for elite soccer. Journal of Sports Science, 18, 669683. 
Bustos-Viviescas et al. Consumo máximo de oxígeno de árbitros colombianos de fútbol

Ruiz Caballero, J. A., Ojeda, E. B., Sarmiento, S., Valdivielso, M. N., García-Manso, J. M., Garcia-Aranda, J. M., Mallo, J., y Helsen, W. (2011). International SportMed Journal, 12(2), 85-91. Recuperado de: http:// hdl.handle.net/10520/EJC48409

Scribano, A. (2007). El proceso de investigación social cualitativo. Buenos Aires, Argentina: Prometeo Libros.

Weston, M., Castagna, C., Impellizzeri, F. M., Bizzini, M., Williams, A. M., y Gregson, W. (2012). Science and Medicine Applied to Soccer Refereeing. Sport Medicine, 42(79), 615--631. doi: https:// doi.org/10.2165/11632360000000000-00000

Whyte, G. P., George, K., Shave, R., Middleton, N., y Nevill, A. M. (2008). Training induced changes in maximum heart rate. International Journal of Sports Medicine, 29, 129-33.
Yanci Irigoyen, J., Reina Vaíllo, R., Granados Domínguez, C., Salinero Martín, J. J. y Los Arcos Larumbe, A. (2014). Valoración y relación de las características antropométricas y la condición física en árbitros de fútbol. Revista Española de Educación Física y Deportes, 406, 15-27. Recuperado de: http://reefd.es/index.php/reefd/ article/view/23http://reefd.es/ index.php/reefd/article/view/23

Zavorsky, G. S. (2000). Evidence and possible mechanisms of altered maximum heart rate with endurance training and tapering. Sports Medicine, 29, 13-26. 